\title{
Matière et manière dans un roman de Nervèze: 'Les Religieuses amours de Florigene et de Meleagre'
}

\section{Nicole Cazauran}

\section{(2) OpenEdition}

1 Journals

\section{Édition électronique}

URL : https://journals.openedition.org/studifrancesi/40132

DOI : 10.4000/studifrancesi.40132

ISSN : 2421-5856

Éditeur

Rosenberg \& Sellier

\section{Édition imprimée}

Date de publication : 1 juillet 2004

Pagination : 19-32

ISSN : 0039-2944

\section{Référence électronique}

Nicole Cazauran, « Matière et manière dans un roman de Nervèze: 'Les Religieuses amours de Florigene et de Meleagre' », Studi Francesi [En ligne], 142 (XLVIII | I) | 2004, mis en ligne le 30 novembre 2015, consulté le 09 septembre 2021. URL : http://journals.openedition.org/studifrancesi/40132 ; DOI : https://doi.org/10.4000/studifrancesi.40132

\section{(c) (i) (9)}

Studi Francesi è distribuita con Licenza Creative Commons Attribuzione - Non commerciale - Non opere derivate 4.0 Internazionale. 


\section{Matière et manière dans un roman de Nervèze: 'Les Religieuses amours de Florigene et de Meleagre'}

Tous les romans de Nervèze sont oubliés depuis longtemps et seuls les historiens de la littérature se soucient de rappeler qu'ils eurent leur vogue tout au début du XVII ${ }^{\text {eme }}$ siècle au point que les dames, paraît-il, les lisaient à l'église en guise de livres d'heures ${ }^{1}$. Ils étaient si brefs que Nervèze put aisément les rassembler sous le titre d'Amours diverses, bien que la diversité, à première vue, y soit certes moins évidente que les ressemblances. Tout se fond, personnages et incidents, dans une prose qui se veut toujours chargée d'ornements. Peut-on, sans trop se lasser, lire de plus près? J'ai tenté la gageure de bonne foi, en tirant du lot, pour la relative singularité de leur sujet, Les Religieuses Amours de Florigene et de Meleagre: c'est le seul roman de ces années-là, Gustave Régnier l'a dit dès $1908^{2}$, qui esquisse le drame vraisemblable d'une vocation forcée.

C'était là, comme bien d'autres, un de ces petits «romans de sentiment» à mille lieues des longs «romans de chevalerie» ${ }^{3}$ répandus par les proses imprimées jusque vers 1560 au moins et dont les Amadis avaient gardé bien des traits ${ }^{4}$. Plus de merveilleux, plus de foisonnement d'aventures entrelacées où des héros multiples ont tour à tour le premier rôle, plus de recul dans un passé lointain et fabuleux. Tout au contraire: dans un monde qui est celui des lecteurs, dans un décor quotidien, des protagonistes qui se ressemblent d'un roman à l'autre par le raffinement de leur conduite et de leur langage, mais que chacun croit pouvoir rencontrer. Qu'elle finisse bien ou mal, c'est toujours l'histoire d'un jeune couple face aux obstacles qui s'opposent à son bonheur. Tous les romanciers à la mode pourraient bien dire comme Nervèze dans son Avis au lecteur de 16025:

J'ai pris encores un coup l'amour pour mon champ de bataille.

Et c'est l'annonce exacte de ces Religieuses Amours qui ne méritent guère leur titre. Pas d'exemple, ici, de ce romanesque dévot analysé par Nancy Oddo et qui viserait à faire coïncider «émotion amoureuse» et «émotion religieuse» pour susciter enfin une réflexion sur le salut ${ }^{6}$. Avec Florigène et Méléagre, on reste sur terre, sans plus. Qu'on en juge: une petite fille de bonne famille mise au couvent, apparemment pour s'instruire, en fait pour prendre le voile; un amour d'enfance réciproque avec un jeune et brillant cousin; un double obstacle: volonté du père qui la veut religieuse et malentendu qu'il provoque pour brouiller les amants; réconciliation, enlèvement

(1) Le Commerce des Nouvelles restably... p. 4: texte cité et analysé par Hubert Carrier, Les Muses guerrières, Paris, Klincksiek, 1996, ch. II, p. 64-67.

(2) G. Reynier, Le Roman sentimental avant «l'Astrée», Paris 1908, ch. X p. 288 et p. 300.

(3) Les «romans de sentiment» sont une des catégories distinguées par G. Reynier. C'est Charles Sorel qui a le premier parlé de «romans de chevalerie»: Bibliothèque françoise, 1644, titre du ch. VIII.

(4) Voir N. Cazauran, «Amadis de Gaule en 1540: un nouveau roman de chevalerie?», Les Ama- dis en France au XVI' ${ }^{\text {eme }}$ siècle, Cabiers V. L. Saulnier 17 pp. 21-39.

(5) Le texte des Religieuses Amours sera cité, sauf mention contraire, dans sa version de 1602

(6) N. Oddo, «Antoine de Nervèze: vieux Protée ou caméléon mondain?», p. 56, Littératures classiques $\mathrm{n}^{\circ} 31$, automne 1997. N. Oddo classe Les Religieuses amours au nombre des «quatre romans dévots» de Nervèze (p. 44-45) et, selon elle, le dénouement heureux se détache «de la spiritualité mais non de la dévotion» (pp. 51). 
en tout bien tout honneur suivi d'un mariage - Méléagre a eu soin d'aller chercher à Rome la dispense libérant Florigène des vœux qu'elle a prononcés. Le couvent n'est là qu'un cadre provisoire et un obstacle à l'accomplissement de l'amour humain. Le récit rebondit par des péripéties vécues dans l'Espagne où les jeunes gens ont fui avant de s'achever pour de bon par un dénouement où s'accordent morale, amour et société.

Surtout, pour Nervèze comme pour d'autres et dans ce roman-là comme ailleurs, l'histoire d'amour paraît bien prétexte à propos sur l'amour. «Parlez-nous d'amour», c'est le titre de l'article d'Yves Giraud sur «Roman et sentiment chez Nervèze» ${ }^{7}$ et, en effet, il s'agit d'en parler et d'en bien parler. Dans ces cent vingt petits feuillets, quasi la moitié est vouée à des discours en forme, à des échanges de lettres en stricte symétrie, voire à des stances respectivement de trente-deux et trente-huit vers. Les deux héros sont, comme il se doit, toujours prêts à briller. Méléagre envoie ses vers «pour faire voir» qu'il est favorisé des Muses (f. 46) et Florigène est capable «de respondre aussi bien aux Poesies, qu'aux lettres de son Amant» (f.49). Rien ne peut leur faire perdre leur éloquence. C'est au point que Méléagre blessé et quasi expirant harangue son épouse pendant trois feuillets recto-verso, en commençant par une période du plus beau style et si longue que j'en cite seulement le début (f. 100):

Que j'ay de regret (chere ame) de voir si tost la fin de nos contentements et qu'il faille qu'un si petit nombre d'années soient les severes limites de notre union...

Quant à Florigène, elle lui répond, nous dit-on, «à mots interrompus», mais il n’y paraît guère (f. 102 v ${ }^{\circ}$ - 103):

Que je te perde, cher espoux, sans me perdre avec toy: que tu meures, mon esperance, et que je te survive...

Le mouvement oratoire se poursuit à loisir jusqu'au «Non, non (mon Meleagre)» qui inaugure la période finale.

Nervèze tire même parti du commun talent, du «bel esprit» qu'il leur a prêté pour agencer l'imbroglio qui réussit un moment à les séparer ${ }^{8:}$ on les prie de prêter leur plume, Florigène à une religieuse qui veut rompre avec un gentilhomme, Méléagre à un gentilhomme qui veut, lui, rompre avec une religieuse. Ni la religieuse, ni le gentilhomme n'existent, mais les deux amants, recevant chacun la lettre écrite par l'autre, la liront comme vraie et se croiront abandonnés. C'est ce que voulait le père de Florigène. Reste que ces deux lettres fictives appartiennent aussi au florilège que le roman prétendait offrir. Dans ces Religieuses Amours abondent, on le voit, les modèles de bien dire que Charles Sorel va bientôt railler sans pitié.

Dans l'espace qui entoure ces morceaux d'apparat et comme dans leurs interstices, Nervèze conduit le récit qui les provoque sans recherche ni complication. Il lui suffit, comme à son ordinaire, de suivre l'ordre des événements, avec une exception pourtant. L'histoire s'ouvre sur le couvent et l'instruction qu'y reçoit la petite Florigène, mais revient un moment en arrière, sur son «enfance», pour introduire son cousin Méléagre et leur attachement réciproque ${ }^{9}$. Arès quoi, tout reprend son cours. La manière propre de Nervèze se reconnaît à sa présence insistante - indiscrète a-t-on

(7) Travaux de littérature VI, 1993, ADIREL, p. 103-124.

(8) Dans un autre article, Y. GIRAud a noté cet emploi comme un «cas intéressant»: «L'insertion épistolaire dans le roman sentimental de Nervèze»,
Les genres insérés dans le Roman, Université Jean Moulin, Actes du colloque de 1992, p. 119.

(9) C'est le lieu d'un plus-que-parfait unique, sauf erreur, dans le texte: «Or l'enfance de ceste créature avoit esté eslevee...»(f. 6). 
dit - dans le rôle de narrateur. Sur ce point encore, il n'est pas isolé en son temps ${ }^{10}$. Mais tout lui est bon pour se faire entendre, apostrophes à ses personnages ou à ses lecteurs, ruptures délibérées pour passer d'un épisode à un autre, commentaires moraux ou affectifs, voire quasi lyriques, et dans cette brève histoire il prend longuement la parole à la première personne, dix fois au moins ${ }^{11}$. Mêlant souci didactique, désinvolture dans les enchaînements, viruosité ostentatoire et parfois subtilité psychologique, il intervient partout et sans cesse, aux dépens de ce qu'il raconte. De grands romanciers l'ont fait volontiers, et même dans des récits assez brefs - témoin Georges Eliot dans Adam Bede. Mais dans Les Religieuses Amours, l'irruption si fréquente du narrateur reste incongrue: la trame est trop mince, les personnages trop pâles pour supporter tous ces suspens qui interrompent et l'histoire et les rôles de ces acteurs.

Somme toute, avec cette complaisance de Nervèze à se mettre en scène comme avec son obstination à déployer toutes les ressources de sa rhétorique, ces Religieuses Amours suffiraient à définir sa manière dans les Amours diverses.

A cette manière de faire, ou plutôt de ne pas faire un roman s'ajoute le fameux «style Nervèze» que Roger Zuber s'est le premier soucié d'expliquer par son contexte $^{12}$, mais qui n'en est pas moins un style où se déploie une écriture contournée, surchargée de figures de toutes sortes au point que le sens, prétendait déjà Charles Sorel en 1664, s'y fait «peu intelligible» ${ }^{13}$. Métaphores filées, anaphores, polynomies, chiasmes,parallélismes et antithèses, oxymores et polyptotes, paronomases... tout se retrouve dans Les Religieuses Amours de l'inventaire qu'a pu faire Yves Giraud, exemples à l'appui ${ }^{14}$. N'insistons pas.

Dans cette surabondance, il y a pourtant quelques beautés éparses qui se verraient mieux si elles se détachaient sur un fond plus uni, tels ces jeunes amants séparés, «confus en leurs resveries et errants parmy les Demons de la solitude» (f. 19) ou bien ces «eslancemens amers» qui troublent Florigène devenue religieuse et découvrant que Méléagre n'était pas infidèle (f. $77 \mathrm{v}^{\circ}$ ). L'on peut même se plaire aux stances où Méléagre reprend assez joliment pour finir des images traditionnelles (f. 47 et $\left.\mathrm{v}^{\circ}\right)$ :

Mes vœux vont costoyant les rives de la mort

Sur la mer de mes pleurs, tes yeux servant d'étoilles

Et pour pousser ma nef à ce tenebreux port,

Mon deuil fournit les vents, et ton voile les voiles...

Les personnages savent à l'occasion user d'une rhétorique ferme et quasi classi-

(10) Sur cet artifice et ses différents usages, voir J. CHUPEAU, «Quelques formes caractéristiques de l'écriture romanesque à la fin du XVI ${ }^{\text {eme }}$ siècle», pp. 223-225, L'Automne de la Renaissance, 1580-1630, Paris, Vrin, 1981. Quand il s'agit de changer le lieu de l'action et de passer d'un épisode à l'autre, les longs romans médiévaux n'hésitaient pas devant ces interventions et l'Amadis de 1540 les pratiquait, plus souvent que ne l'a dit J. Chupeau (voir ch. IV, XVI, XXI...). Sur les irruptions du narrateur voir aussi Y. GIRAUD, article cité note 7, p. 118-119 et Gabrielle VERDIER, «Lire Nervèze? Rhetoric and Readability in Early Seventeenth Century Narrative», Papers on french seventeenth century litterature, XII, 1985, Seattle, p. 436.

(11) Exemples: f. $24 v^{\circ}$, f. $25 v^{\circ}-27$, f. $34 v^{\circ}$ 35, (console toi Meleagre); f. 40 (Mais sautons... et voyons...) ; f. $45-46$ (qu'avez-vous dict ma plume... Retournons à nostre Meleagre); f. 75 -75 $\mathrm{v}^{\circ}$; f. $80-81 \mathrm{v}^{\circ} ; 83 \mathrm{v}^{\circ}$; f. $90-90 \mathrm{v}^{\circ}$; f. 99 ; f. 115 $v^{\circ}-116$.

(12) R. ZuBER, «Grandeur et misère du style Nervèze», L' Automne de la Renaissance..., pp. 53 - 64. F. BRunot lui-même, si loin de goûter l'abus des expressions figurées, multiplie les exemples pour montrer que cet abus n'est pas propre à Nervèze: Histoire de la langue française, t. III, rééd. 1966, 1. II, ch. IX p. 243-245.

(13) La Bibliothèque Françoise, Paris, La Compagnie des libraires du Palais, «Du Progrez de la Langue Françoise» p. 232. R. ZuBER a souligné la mauvaise foi des polémiques contre Nervèze en étudiant le discrédit de son style (art. cit note 12).

(14) Y. GIRAUD, article cité note 7, p. 119 - 120 et notes. 
que. Ainsi Florigène pour clore la lettre de rupture qu'elle croit écrire au nom d'une religieuse (f. 65):

[J'] implore les douces rigueurs de vostre oubly et du silence pour le repos de celle qui se sépare de vous pour s'unir à Dieu et estre à elle mesme.

Ou bien l'oncle de Méléagre pour achever ses remontrances (f. $113 \mathrm{v}^{\circ}$-114):

Rentrez en vous-mesme, mon nepveu et vous en venez à moy: donnez ce contentement à vostre mere et ne permettez pas que vostre rencontre soit inutile à son esperance et au desir que j'ay que vous possediez vos biens, vos honneurs et les grandeurs de vostre race.

Mais il y a bien plus surprenant que ces bonheurs d'expression où le hasard fut peut-être pour quelque chose: ce sont les variantes qui viennent modifier le texte, non seulement quand l'histoire, désormais précédée d'un argument, est réunie à d'autres dans les Amours diverses de 1605, mais encore quand l'ensemble est repris et complété en $1611^{15}$. Et ces variantes ont de quoi déconcerter le lecteur accoutumé aux recherches du «style Nervèze».

Des amplifications, des ornements surajoutés, il n'y en a guère: quatre en 1605, deux de plus en 1611, et qui n'excèdent pas quelques lignes. Rien d'ostentatoire, sinon par deux fois le malencontreux rappel du Méléagre grec et de sa légende: à sa naissance, une des Parques, prenant un tison, aurait prédit qu'il vivrait aussi longtemps que le tison pourrait brûler et ce tison vient figurer les flammes dont le moderne Méléagre brûle pour Florigène (1602, f. 75 et 1605 f. 342 v $^{\circ}$ et 343) et ce nouveau Méléagre, mourant, oppose la tendresse de sa mère à la cruauté d'Althée mère du Méléagre antique $\left(1602\right.$, f. $106 \mathrm{v}^{\circ}$, et 1605, f. 353) ${ }^{16}$. Le reste se fond aisément dans la version première. En revanche, si Nervèze ajoute peu, il s'obstine à réviser son texte dans le détail.

Dès 1605, il corrige des bévues: Florigène maîtrise désormais son mal de mer «comme si la mer l'eust moins tourmentée que son amant» (f. $346 \mathrm{v}^{\circ}$ ) et non pas «comme si la mer l'eust moins espargnee...» (f. 86) ${ }^{17}$. Surtout, il se soucie d'alléger, de préciser, voire de simplifier. En 1605 et encore en 1611, il coupe des formules redondantes, inutiles, souvent inscrites entre parenthèses: les discours de Florigène ne seront plus «ornement de son mérite» (f. 6; 1605, f. 319 v) ni ses soupirs «fils de la douleur (f. $\left.21 \mathrm{v}^{\circ} ; 1605, \mathrm{f} .324 \mathrm{v}^{\circ}\right) »$ ni la ville de Paris «l'abrégé de nostre France» (f. $73 \mathrm{v}^{\circ} ; 1605$, f. 342). Il sait aussi changer un mot pour un autre qui convient mieux. Quand il s'agit de la «procedure» contre un seigneur espagnol qui a insulté et attaqué Méléagre, il passe, écrit-il en 1602, «pour ne mesler un procez-verbal» à son «histoire» (f. 99); en 1611, ce sera «pour ne mesler un procez criminel à une histoire d'amour» (f. 144). Dans ces retouches ponctuelles, il est toujours attentif au contexte, se gardant qu'un mot nouveau vienne en répéter un déjà employé. En 1611, Florigène ne se plaint plus d'être au couvent «soubs l'auctorité d'une loy sauvage» mais, avec plus de retenue, sous celle «d'une loy forcée» et, du coup, elle ne parle plus des «liens forcez» de sa liberté, mais des «liens solitaires» $\left(1602, \mathrm{f} .11 ; 1611 \mathrm{f} .103 \mathrm{v}^{\circ}\right)$. Enfin, s'il arrive à Nervèze de récrire apparemment pour embellir - ainsi au début de stances de

(15) Voir, en appendice, le texte de l'argument p. 30 et pp. $29-31$ sur les éditions de 1605 et 1611 et leurs reprises, Chaque histoire, désignée par sa place dans la série, n'a plus de titre particulier sauf en 1617 .

(16) La fable 171 d'Hygin donne une version très proche de la première amplification, ainsi introduite par Nervèze: «Les fables font mention d'un Méléagre fils de Daeneus...» (pour Oeneus). La fable 174 raconte la mort de Méléagre.

(17) Parfois, il s'agissait, semble-t-il, de simples coquilles: les Dieux et les hommes de l'antiquité le «fourniroient» d'exemples, lit-on en 1605 (f. 329) et non plus le «formeroient» $\left(1602\right.$-f. $\left.35 \mathrm{v}^{\circ}\right)$. 
Méléagre (f. 46 et $v^{\circ} ; 1611$, f $127 v^{\circ}$ ) - c'est de simplifier qu'il se soucie plus volontiers. Dès 1605, il renonce à évoquer des esprits «amoureusement passionnez au service du Sauveur et passionnément amoureux de sa miséricorde»: il garde le premier terme seulement et ajoute «et désireux de sa miséricorde» (1602, f. 3; 1605, f. 318 vº). En 1611, Méléagre ne parle plus de la «discrette tombe», mais du «discret silence» où il garde le souvenir des mots qui le liaient à Florigène enfant (f. 14; 1661, f. $104 \mathrm{v}^{\circ}$ ). Et l'amour d'enfance de Florigène, ses «jeunes feux» ne sont plus, bizarrement, des «glaces errantes» (f. 15), mais des «estoiles errantes» (1611, f. 105). Plus tard, elle ne dit plus à sa nourrice $\left(\mathrm{f} .55 \mathrm{v}^{\circ}\right)$ :

Vostre fidelité se jette à corps perdu sur mes sanglots...

Mais tout bonnement (1611, f. 124):

Vostre fidelité s'offre à moy...

L'éloge de Henri de Navarre était bien contourné dans la première version (f. 73):

Sa prudente valeur coupoit les oliviers de ses victoires pour donner des rameaux de paix aux siens...

L'image devient plus claire en se dédoublant (1605, f. 342):

Sa prudente valeur joignoit l'olivier de sa clemence aux lauriers de sa victoire...

Parfois une phrase est à deux reprises corrigée et simplifiée. Nervèze, pour blâmer un père de famille qui préserve la fortune de sa «maison» en mettant en religion certains de ses enfants, avait d'abord écrit (f. 26):

Il cherche sous la faveur des contraintes les deplorables remedes pour contenter l'avarice et luy donne un masque de devotion comme si Dieu ne voyoit point au delà des apparences.

Dès 1605 (f. 326) il coupe «sous la faveur des contraintes» et en 1611 (f. 110) il retouche tout le début: votion...

Il cherche des moyens pour contenter son avarice à laquelle il donne un masque de de-

Images et figures un peu plus rares et souvent moins forcées ou incongrues, telles sont les retouches qu'il pratique dès 1605 et plus encore en 1611.

Ces retouches qu'il apporte ne modifient ni le sens ou l'ampleur de l'histoire ni la figure des personnages: on comprend qu'elles n'aient jamais retenu l'attention ${ }^{18}$ et que les histoires des Amours diverses soient citées indifféremment dans les éditions de 1606 (1605 étant rare), 1608, 1611, 1621, ou, plus rarement, dans leur version première. A en croire l'exemple des Religieuses Amours, elles importent pourtant. En les considérant, on n'est pas seulement porté à plus d'indulgence et à goûter quelques réussites dans ce fameux style Nervèze qui se démoda si vite. On découvre un Ner-

(18) C'est au point que D. Bungs, étudiant Les ouvres romanesques d'Antoine de Nervèze (Microfilm, New-York, 1972), n'en dit pas un mot dans les soixante pages de son chapitre IV consacré au style.
Ainsi, p. 110, elle cite comme exemple de métaphore, d'après son édition de référence (1606), une formule venant de la première version et supprimée en 1611: voir supra p. 6 «Vostre fidelité...» etc. 
vèze attentif à son propre texte, un auteur qui était en droit de faire mettre au titre des Amours diverses en 1605 ou 1606 et encore en 1611, «revues et corrigees» - et le privilège de 1605 , repris ensuite, insistait «nouvellement reveuës, corrigees et augmentees par lui-même». Il revoit, il corrige en effet et dans ces variantes qui paraissent plus souvent des repentirs que de nouveaux ornements, on est tenté de trouver une cohérence et le signe d'un travail concerté. Ce Nervèze dont le nom devint, sous la plume du père Bouhours, synonyme de «galimatias», lui que le terrible Charles Sorel accusait d'avoir cherché l'élégance par des «métaphores continuelles et d'autres figures le plus souvent si contraintes que le sens en estoit extravagant ou peu intelligible ${ }^{19} \gg$, n'a pas cessé d'être pour lui-même un lecteur critique, capable d'élaguer et de simplifier. Conclusion qu'il faudrait confirmer par l'examen de ses autres histoires, et qu'il faut à tout le moins tempérer en ajoutant qu'il s'est arrêté en si bon chemin, mais conclusion paradoxale face à sa réputation. On doit admettre qu'il a su, à l'occasion, renoncer à telles ou telles fleurs de rhétorique prodiguées hors propos pour leur préférer des tournures moins apprêtées et plus claires ${ }^{20}$. Qui l'eût cru?

Et le roman? Tant de propos sur l'amour lui laissent - ils quelque place dans les Religieuses Amours? C'est une des deux histoires retenues par Yves Giraud pour étudier «Roman et sentiment chez Nervèze», mais il n'hésitait pas à conclure ${ }^{21}$ :

Les histoires de Nervèze ne sont pas intéressantes parce que le roman ne l'intéresse pas.

C'est peut être aller un peu vite en besogne. Certes, dans le flot éloquent des paroles, lettres, discours, dans les gloses qui s'y mêlent, on risque bien de perdre de vue l'action et ses personnages. Il y a pourtant une action et des personnages, ou du moins des silhouettes assez distinctes.

L'action est précisément située dans le lieu et le temps. Le couvent est tout proche d'une rivière quasi à son estuaire puisque s'y fait sentir le «reflux» de l'Océan (f. 2). La navigation des amants qui fuient la France les conduit à Séville, mais ils ont «mouillé l'ancre» en l' île de Cadix (f. $115 \mathrm{v}^{\circ}$ ), comme fait à la fin le pilote de la mère de Méléagre «pour prendre le vent» qui doit lui «ouvrir le passage de la rivière» menant à Séville (f. 116). Quant au temps, c'est très précisément celui où vit Nervèze et même où il publie son histoire. Il écrit, dit-il, «soubs le sauf conduit de la paix» mais son «suject» a pris naissance dans les «divisions civiles»f. $1 v^{\circ}$. En effet, son jeune héros, après sa rupture provisoire avec Florigène, rejoint les troupes de Henri de Navarre «lequel en ce temps (...) contestoit peniblement avec ses subjects l'héritage de ses pères» et même il se retrouve au siège de Paris, donc au printemps ou à l'été 1590 (f. 73 et $v^{\circ}$ ). Quand les amants ont fui à Séville et s'y sont mariés, ils vivent quelques années heureuses avant les ultimes péripéties que plusieurs détails situent à l'automne 1598. Le texte insiste sur la paix rétablie entre la France et l'Espagne, et le traité de Vervins fut conclu le 2 mai 1598. L'oncle de Méléagre, nous dit-on, est venu jusqu'à Lisbonne «pour voir le pays soubs l'asseurance» de cette paix $\left(\mathrm{f} .111 \mathrm{v}^{\circ}\right)$ et Méléagre craint de rencontrer dans les villes «des François de sa cognoisssance, à ceste heure que la paix et les curiositez les promenoient surement par les Espagnes»(f. $\left.115 \mathrm{v}^{\circ}\right)$. Quant au roi d'Espagne qui joue un rôle dans le récit, il est tout au début de son

(19) D. Bouhours, Entretiens d'Ariste et d'Eugène (Paris, S. Marbre Cramoisy, 1671), La langue françoise, second entretien, p. 128 «Le nervèze, le galimatias et le phébus ne reviendront poinct...»; CH. SOREL, op. cit. note 13 , ibid.

(20) Ce souci est perceptible jusque dans les retouches qu'il apporte à son éloge de Sully dans l'épître dédicatoire des Amours diverses entre 1606 et 1611 .

(21) Y. GIRAUD, article cité note 7, p. 122. Il ajoute: «ce qui ne veut pas dire qu'il en ignore les procédés». 
règne. Florigène, dans l'édition de 1602, lui parle de ses «naissantes couronnes» (f. $98 \mathrm{v}^{\circ}$ ) et l'adjectif disparaît en 1611(f. $143 \mathrm{v}^{\circ}$ ) par une coupure imperceptible qui atteste l'attention portée au détail du texte. Avant qu'il réponde, on rappelle encore qu'il est «au commencement de sa dignité Monarchique» et «sur un traicté de paix avec le Roy de France» (f. 99). Autant d'indices qui désignent précisément Philippe III dont le père est mort, après le traité de Vervins, le 13 septembre 1598. La chronologie de l'action indique ainsi sans doute possible que le roman fut écrit après le privilège du 27 juin 1598 accordé à Anthoine du Brueil pour imprimer Les Euvres du Sieur de Nervèze, mais peut-être aussi avant que renaissent plus vivement les tensions entre la France et l'Espagne, dès 1601. A coup sûr, elle inscrit l'histoire dans l'actualité que connaissent, que vivent ses premiers lecteurs. Rien d'original, à première vue, dans tout cela. Il s'agit pour Nervèze, comme pour les autres romanciers du temps, de se distinguer des romans médiévaux et des Amadis avec leur décor et leur passé pareillement fabuleux. Encore a-t-il eu soin d'introduire une minutieuse précision dans cet arrière-plan.

Dans ces lieux connus, dans ce temps si proche, les deux amants sont séparés par le stratagème que mettent en œuvre la gouvernante et le père de Florigène. Ce sont les lettres fictives dont j'ai déjà parlé et si l'on peut s'étonner que ni Florigène ni Méléagre ne s'étonnent, eux, d'avoir à écrire une lettre pour rompre la liaison d'une religieuse et d'un gentilhomme, l'acheminement des deux fausses lettres s'explique aisément: on est loin des complications arbitraires et risibles inventées par Boursault en 1670, dans Artémise et Poliante, autour de deux lettres, vraies celle-là, égarées par inadvertance et qui vont, elles aussi, séparer un moment les héros ${ }^{22}$. Après la réconciliation et l'enlèvement, Florigène, sur le bateau, rêve poursuite et naufrage, mais ce n'est qu'un rêve, et si des corsaires anglais sont en vue, ils restent au loin. A Séville même, pas de rival machiavélique obstiné à séduire et manœuvrant dans l'ombre, mais seulement, pour amener la catastrophe, une scène de rue convenant au décor. Méléagre et Florigène un soir «prennent le frais (...) le long de la rivière», quand un marquis castillan aborde Florigène puis, «se laissant surprendre à ceste beauté et surmonter à l'insolence», en vient à offenser le mari «de la langue et de l'espée»: d'où un duel où Méléagre, lui-même blessé, blesse gravement l'espagnol et tue un de ses serviteurs, avant d'être emprisonné (f. 93 à $94 \mathrm{v}^{\circ}$ ) et enfin libéré sur ordre du roi d'Espagne à qui Florigène est allée demander justice. La péripétie, en ces temps où les gentilshommes tirent facilement l'épée, n'a rien qui puisse surprendre et elle s'achève sur une enquête et un procès en bonne et due forme (f. 97 à $99 \mathrm{v}^{\circ}$ ). Les excès du romanesque ne se voient guère que dans la soudaine guérison de Méléagre mourant que la Mort elle-même, émue par les plaintes des deux époux, décide d'épargner (f. 110). Encore n'est-ce là, à tout prendre, que manière de parler.

(22) Selon H. Coulet (Le Roman avant la Révolution, Paris, Colin, 1967, ch. IV, p. 143), Boursault pour Artémis et Poliante aurait emprunté «à peu près toute la matière» aux Religieuses Amours de Nervèze. C'est beaucoup dire. On trouve dans sa «nouvelle» l'histoire d'un amour d'enfance - mais favorisé par l'amitié qui lie les jeunes parents ; d'une séparation, mais voulue provisoire, la petite fille au couvent où sa tante est abbesse, le jeune garçon au collège de La Flèche ; et enfin d'un malentendu à propos des lettres, mais provoqué par deux mensonges improvisés et disjoints bien que semblables. Une première lettre de Poliante à Artémise doit lui être remise par sa mère qui commence par la faire admirer si bien qu'elle passe de main en main et s'égare: la mère de Poliante lui affirme qu'Artemise l'a bien reçue et ne l'aime plus puisqu'elle ne lui répond pas. Artémise six mois après écrit à Poliante et confie sa lettre à sa propre mère qui la montre au père du jeune homme qui la perd lui aussi: c'est au tour de la mère d'Artémise de prétendre que Poliante a bien reçu la lettre et ne se soucie pas de répondre. Désolation d'Artémise qui se fait novice, maladie du jeune Poliante que son père tire du collège en lui révélant - enfin - qu'Artémise lui a écrit. Après quoi l'action diverge radicalement: pas d'enlèvement, c'est son père qui tire Artémise du couvent, puis la marie à Poliante alors qu'elle est courtisée par un grand prince. Et il y a encore toute une suite où Artémise déguisée en jeune officier suit incognito son mari à la guerre, manque de mourir au siège de Lille en se précipitant à son secours, avant la reconnaissance qui achève leurs aventures. 
Après quoi vient un dénouement où tout s'arrange pour le mieux avec une facilité déconcertante et dont on peut sourire, mais où les coïncidences sont toujours justifiées: si le couple, avec son enfant, s'en va à Lisbonne, c'est qu'une épidémie sévit à Séville (f. 111); si Méléagre y rencontre son oncle sous qui il avait été lieutenant au siège de Paris (f. 74), c'est que «l'asseurance de la paix» entre la France et l'Espagne invite à voyager (f. $111 \mathrm{v}^{\circ}$ ) et s'il s'en va dans l'île de Cadix au lieu de retourner à Séville, c'est pour éviter d'autres rencontres (f. $115 \mathrm{v}^{\circ}$ ); enfin, si sa mère, partie pour le chercher à Séville où, au dire de l'oncle, il doit se rendre, aborde à Cadix, c'est que les navires doivent y mouiller pour prendre le vent. Tous les incidents, on doit le reconnaître, sont agencés avec soin.

Autour de Méléagre et Florigène, des personnages secondaires qui paraissent plus divers et plus précisément esquissés que les comparses réduits à leur fonction dont se contentent d'ordinaire les auteurs des petits récits sentimentaux. Ici chacun ou presque a ses traits propres. Rosemonde qui a nourri Florigène et la sert «en qualité de gouvernante» (f. $21 \mathrm{v}^{\circ}$ ) lui est toute dévouée, s'inquiète sincèrement en la voyant pleurer «en ses resveries ordinaires» (f. $52 \mathrm{v}^{\circ}$ ) et ne trahit sa confiance que par souci de son «renom», sûre que ses passions ne cèderont pas à une «honorable remonstrance» (f. 57) - d'où le stratagème des lettres qu'elle invente et le rôle qu'elle y joue. L'oncle de Méléagre profite de la paix «pour voir le pays» et quand il rencontre son neveu, s'abstenant des reproches, il lui tient un discours plein de bon sens: il l'exhorte à se détacher des «folles passions» et à rentrer en France pour contenter sa mère, et retrouver ses «biens», ses honneurs» et «les grandeurs» de sa race (f. 112114). Jusqu'au serviteur de Méléagre qui a sa physionomie: il ne révèle à la mère du héros le logis du couple que «vaincu par le respect ancien qu'il porte à ceste vieille Damoiselle» (f. $\left.116 \mathrm{v}^{\circ}\right)$.

Enfin, dans ce drame d'une vocation forcée, Nervèze s'est gardé de durcir le trait. L'abbesse règne sur un couvent qui attire «les plus belles ames de la région» (f. 2) et la piété n'y est pas affectation. Quoi qu'on en ait dit, ${ }^{23}$ cet éloge initial n'est pas démenti par les scènes qui s'y déroulent. Florigène y apprend «la modestie» comme la musique et l'abbesse sait lui faire «chanter et jouer quelques airs spirituels» (f. 5 et v ) pour recréer les esprits en dehors des moments d'oraison. Au moment où Florigène, à la demande de son père, devrait prononcer ses vœux, cette même abbesse se garde de peser sur la décision de la jeune fille. Elle le souhaite aussi, mais «pourveu - lui dit-elle - que vostre intention s'y rapporte» (f. $31 \mathrm{v}^{\circ}$ ). Et quand Florigène a demandé un délai pour se résoudre, elle est prête aussitôt à l'accorder: elle «ne veut point que les devotions forcees entrent dans son cloistre» ${ }^{24}$. Quant au rôle du père, il est bien moins noir que dans d'autres romans contemporains où les parents contraignent leur fille par cupidité ou par vanité à des mariages qui leur répugnent. Certes, il ment à Florigène en lui faisant croire qu'il la met au couvent seulement pour quatre ou cinq ans $\left(\mathrm{f} .3 \mathrm{v}^{\circ} 4\right)$ et Nervèze a soin, en le condamnant, d'en faire l'exemple des pères qui abusent de leur autorité paternelle sous «le masque de dévotion» et rendent leurs enfants «misérables» pour maintenir leur maison (f. $25 \mathrm{v}$. - $26 \mathrm{v}^{\circ}$ ). Mais il a commencé par expliquer les motifs de cette coupable «prudence»: ce père est âgé, il a plusieurs filles, peu de moyens pour les «bien pourvoir» et «les mettre à leur aise» avant de mourir (f. 25). Quand il revoit Florigène d'enfant devenue jeune fille, il admire son «bel esprit» et quand il l'entend chanter, il est sur le point de renoncer à son projet (f. 27-29):

(23) Voir G. VERDIER, article cité n. 10, p. 436, qui y voit une amplification oratoire en contradiction avec sa fonction de couvent «prison».
(24) Version de 1611, f. $113 \mathrm{v}^{\circ}$; version première, f. 34 «que les devotions forcees habitent ses autels». 
...Ce bon vieillard de pere l'estimant la plus belle rose de sa lignee fuct sur le poinct de violer la promesse qu'il avoict faite à Dieu.

Il rentre aussitôt, il est vrai, dans son rôle convenu et ne sera pas touché par la lettre où Florigène l'implore de lui laisser sa liberté (f. 59):

Car tirer une fille du monde contre son gré c'est se repentir de l'avoir fait naistre.

Il n'hésitera pas à tenir sa partie dans le stratagème des lettres trompeuses inventé par la gouvernante. Après quoi, il sort du récit et on apprend incidemment, tout à la fin, qu'il est mort. C'est un rôle épisodique qui lui est dévolu, essentiel au nœud et à la première péripétie, mais il en reste l'image d'un père tout ordinaire, pas plus mauvais qu'un autre, agissant sans beaucoup hésiter comme on fait autour de lui et pourtant sensible au charme de sa fille. La mère de Méléagre, elle, ne sort pas de la pénombre avant le dénouement. Mais quelques touches esquissent auparavant la silhouette d'une mère aimante et dévouée à son enfant: elle est veuve avec, pour compagnie, ce fils qu'elle élève (f. $\left.6 \mathrm{v}^{\circ}\right)$; quand il lui fait croire qu'il la quitte pour aller lever quelques troupes destinées au Roi, elle le laisse partir «avec une bonne somme d'argent» en se réjouissant de cette «honorable entreprise»; comme il convient à une bonne mère, elle est «jalouse de la fortune de son fils» (f. 82); après sa fuite, elle vit dans les «fascheries», attendant son retour «pour mourir après contente» (f. 112). Ainsi, tout est en place pour les retrouvailles finales, dans le goût de Diderot, où la «bonne dame» (f.116), avec larmes et pardons, bénit le jeune couple et prend dans ses bras pour le chérir le petit-fils qu'on lui présente (f. 117 et $\mathrm{v}^{\circ}$ ).

A ce qui peut paraître, somme toute, un récit d'un romanesque moyen, s'ajoutent parfois des précisions chronologiques qui importent à la conduite des personnages. Le couvent où Florigène est placée - «fort jeune» note l'Argument de 1605 - n'est pas seulement, n'est pas d'abord pour elle le lieu où elle est condamnée à se vouer à Dieu sans en avoir la vocation. Elle a dix ans quand elle y entre, pour quatre ou cinq ans lui dit-on, et son «naturel (...) ployable à toutes impressions» (f. 4) s'en accommode patiemment. Elle prend le voile blanc des novices sans y voir un engagement. Dans ce couvent réputé la petite Florigène acquiert les «perfections» propres à illustrer sa beauté (f. 5):

Cependant elle façonne son esprit aux leçons de la modestie, se rend capable de plusieurs beaux exercices que l'art luy enseignoit, comme la musique et la science du luth ${ }^{25}$.

En grandissant, elle se distingue alors par «son luth, sa voix et ses discours» et si bien qu'elle est nommée «le soleil de ceste compagnie religieuse», mais, pour achever le portrait, le narrateur précise que «ses jeunes ardeurs» ont encore «quelque intelligence avec le monde» (f. 6). Ainsi s'introduit sans rupture un retour en arrière où Méléagre surgit aux côtés de Florigène dans le bref tableau d'un amour d'enfance entre cousins:élevés ensemble chez la mère de Méléagre, ils se promettent dans leurs «puériles feux»(f. $\left.7 v^{\circ}\right)$ qu'ils seront mari et femme. Puis l'on revient au séjour de Florigène au couvent et Nervèze montre le jeune Méléagre répétant au père de Florigène qu'ils se sont promis mariage, «discours» que le père prend «en risée comme d'un jeune homme» (f. $\left.7 \mathrm{v}^{\circ}\right)$.

Même insistance sur le temps et sur l'âge des jeunes héros quand Méléagre fait visite à Florigène dans son couvent. Ils sont conscients l'un et l'autre d'avoir quitté

(25) Insistance plus grande en 1611 (f. 110): nette et du luth». «comme de chanter la musique et jouer de l'espi- 
l'enfance, d'être dans leur «adolescence» (f. 10) - le mot y est - sans avoir oublié leurs «premiers desseins» et Méléagre avoue que «la façon retenue» de Florigène, sa «beauté formée» lui ont d'abord imposé une «discretion», une «sage timidité» qu'il n'avait pas au temps où la «simplicité et naïveté» la gouvernaient (f. 15-16). Quand il écrit ensuite à sa «belle novice», c'est l'occasion pour Florigène de s'affliger, de se prendre en pitié elle-même «lisant les traits de sa condition, representée par celuy qui la regrette» (f. 21 et $\mathrm{v}^{\circ}$ ): subtile notation qui convient à une toute jeune fille, peu portée encore, à examiner sa position. Et quand son père vient au couvent pour lui faire prononcer ses vœux, c'est bien une jeune fille qu'il découvre au lieu de l'enfant qu'il avait quittée (f. 27):

Elle paraist devant son pere toute autre qu'il ne l'avoit veûe, de geste et de suffisance.

Plus loin, on nous précise que «cinq ans» avaient passé (f. $59 \mathrm{v}^{\circ}$ ). L'héroïne a donc désormais quinze ans et son amant sans doute un peu plus. Leur jeunesse importe encore au récit au moment du malentendu provoqué pour les séparer. Méléagre «se desrobbe peu à peu de sa passion» et, devenu lieutenant dans une compagnie commandée par son oncle, il joint la «suffisance»» à la «jeunesse» et cette «jeunesse» rend «ses actions plus admirables» (f. $\left.72 \mathrm{v}^{\circ}-74\right)$. Quant à Florigène, qui s'est décidée à prendre le voile, elle commence «à se moquer de ses erreurs passées», réconciliant en elle «jeunesse» et «prudence» (f. $75 \mathrm{v}^{\circ}$ ), prête à dire son «dernier adieu» à la mondanité. Pour qu' elle sente un «divorce en ses imaginations» (f. 77), il faut que sa gouvernante, trop sûre qu'elle est désormais toute à Dieu, se vante de son stratagème.

Amour d'enfance et de jeunesse donc ${ }^{27}$, un moment suspendu, mais prompt à reprendre. Et ce sont deux jeunes amants qui s'enfuient ensemble et que Nervèze nous montre, sur le navire qui les emporte, dans les bras l'un de l'autre, notant, avec une précision insolite chez lui, comment leurs baisers «alterent le desir» et comment leur «continence» triomphe de leur «sensualité», tandis qu'ils «rodent autour de leur felicité» (f. $\left.91 \mathrm{v}^{\circ}\right)^{28}$. L'oncle de Méléagre, quand il rencontre le jeune homme quelques années plus tard, saura bien lui dire qu'il n'a plus l'âge désormais de tels «coups d'enfance» que furent sa fuite et sa vie incognito en exil (f. 118). Les sentiments et l'aventure du couple sont ainsi pris dans la durée et c'est par là peut-être que ces Religieuses Amours prennent par endroits quelque épaisseur romanesque.

Tout est bien qui finit bien dans ces Religieuses Amours et ce n'est pas si fréquent dans les romans du temps où les malheurs des jeunes amants s'achèvent souvent par la mort de l'un ou de l'autre. Nervèze n'a pas manqué d'y déployer tous les artifices qui firent son renom avant de le rendre bientôt ridicule aux yeux des honnêtes gens - tout juste bon, fera dire Sorel à son Francion, à fournir à un laquais amoureux d'une servante des modèles de «complimens» ${ }^{29}$. Il n'empêche: il a su pourtant, sans que

(26) Dans la version première: «l'expérience» (f. $\left.73 \mathrm{v}^{\circ}\right)$. Le mot ne convenait pas.

(27) A en croire Nervèze dans sa dédicace de l'édition de 1602 «A Madamoiselle S. D. A. M.», il y a peut-être là le souvenir d'un amour d'enfance vécu dans une même terre et que le temps ni l'absence n'ont pu détruire - d'autant que la préface des Amours diverses (1605 et 1611) prétend que ses œuvres profanes lui ont donné le plaisir d'exprimer ses peines «soubs des noms empruntez et des voix supposees auxquelles - dit-il - je faisois dire et souspirer les propres ressentimens de mon âme».
(28) Y. GIRAUD, art. cité note 7, p. 112-113, a noté l'intérêt d'un passage «exceptionnel» dans les romans de Nervèze, alors que ses vers profanes - Essais poetiques de 1605 - ont plusieurs pièces sensuelles: invitations à la volupté et évocation des ébats amoureux. Voir les exemples qu'il en donne dans «La poésie d'Antoine de Nervèze», Mélanges... Louis Terreaux, Paris, Champion, 1994, p. 385-388.

(29) Ch. Sorel, La Vraye Histoire comique de Francion, Paris, P. Billaine, 1663, 1. XI, p. 843-844. Edition A. ADAM, Romanciers du XVII ${ }^{\text {eme }}$ siècle, Bibl. de la Pléiade, p. 432. 
personne lui en sache gré ou même s'en aperçoive, se relire d'un œil critique, attentif à modérer les falbalas de son trop fameux style. Mieux encore, et qui n'exige pas, pour être vu, de patientes allées et venues d'une édition à l'autre, Nervèze narrateur fait preuve ici de quelque adresse. Pour cette histoire de vocation forcée qui faisait, plus qu'aucune autre dans ces Amours diverses, un sujet «prenant et vrai» ${ }^{30}$ (ce sont les mots d'Henri Coulet, si bon connaisseur en matière de roman), il s'est refusé à outrer le pathétique, à multiplier les aventures hors du commun ou même à isoler ses touchantes victimes, au milieu de fantoches, face à des ennemis tout uniment méchants, et il ne laisse pas oublier la jeunesse de ses héros ni même, à l'arrière-plan, le rôle du temps qui passe. On peut se croire dans le monde comme il est et, sans trop prêter au texte, un lecteur de bonne volonté saurait découvrir là comme le fantôme d'un roman, bien pâle assurément, mais assez précisément esquissé pour rester dans la mémoire.

NICOLE CAZAURAN

\section{Appendice: Note sur les exemplaires examinés}

La succession des privilèges et les cessions successives, consenties par A. du Brueil attestent le succès des romans de Nervèze.

1602 Ars. B L 21060

Les Religieuses Amours de Florigene et de Méléagre

par A. de Nervèze. Dernière édition. Paris, Anthoine du Brueil, 1602. In -12

. A. Madamoiselle S.D.A.M.

. Au Lecteur.

. Pièces liminaires:

Sonnet - signé L'Avizé

Quatrain - signé D'Armanthie

Sonnet - signé La Ruze

A Monsieur de Nervèze - signé Adam, secrétaire de

Madame de Mortemart

. Texte -119 folios

A Monsieur de Nervèze - Stances - signé C. D. M.

. Privilège du Roi à Anthoine du Brueil... «d'imprimer Les CEuvres du

Sieur de Nervèze» pour dix ans; donné à Paris le 27 juin 1598.

. Achevé d'imprimer: 3 octobre 1602.

Cette édition de 1602 est ici - sauf mention contraire - le texte de référence. Cette «dernière édition» fait supposer une édition antérieure, mais nécessairement pas avant le début de 1599 (voir supra p. 23-24).

(30) H. Coulet, op. cit. note 22, ch. IV, p. 143 ; Gustave Reynier, dès 1908, insistait aussi sur l'in- térêt, et même sur l'audace du sujet: $o p$. cit. note 2, p. 300 . 


\section{Les Amours diverses. Sept Histoires}

1605 Poitiers - Bibl. mun. - D M 135

Les Amours diverses divisées en sept Histoires

Par le sieur de Nerveze conseiller et secretaire de Monsieur le Prince de Condé.

Reveuës et augmentees.

Paris, Anthoine du Brueil, 1605.

in 12 - $400 \mathrm{f}$., Table et Privilège

Privilège du Roi: «permis à Anthoine du Brueil d'imprimer ou faire imprimer Les CEuvres du Sieur de Nerveze nouvellement reveuës et augmentées par luy mesme». Privilège pour dix ans, daté du 11mars 1605.

- L'ensemble est précédé d'une préface et d'un Sonnet, L'autheur à son livre (v. 1-3): «Enfans de mes labeurs mon plus doux exercice / Ou mon esprit souloit recueillir ses plaisirs / Du temps que vous chantiez mes amoureux souspirs...» Plus de titre particulier pour chaque histoire, mais un «Argument».

- Sixiesme histoire: la dédicace des Religieuses Amours, l'avis Au lecteur et les pièces liminaires ont disparu.

Argument: f. 317 et v $\mathrm{v}^{\circ}$ Texte f. $318-357 \mathrm{v}^{\circ}$.

- Texte de l'Argument, ensuite repris sans variantes.

«Florigene ayant esté mise fort jeune dans un monastere sous pretexte qu'elle n'y seroit que quelques années et enfin son Pere luy ayant descouvert le dessein qu'il avoit de l'arrester en la profession de religieuse: Elle dissimule accortement le regret qu'elle avoit de se voir ainsi trompée en ses esperances et l'amour qu'elle nourrissait dès longtemps pour Meleagre: leurs entreveues: leurs discours amoureux: la prudente invention qu'elle eut pour s'empescher d'être professe, lors que son père l'alla veoir pour ce suject: quelle fut la malicieuse subtilité de Rozemonde sa gouvernante pour diviser ces Amants: le voyage que Meleagre fit à Rome pour avoir les dispenses du mariage de luy et de Florigene: leur fuitte en Espagne, où ils reçoyvent de cruellles secousses de la fortune. Le rencontre (f. $317 \mathrm{v}^{\circ}$ ) d'un oncle de cest Amant à Lisbonne en Portugal: la remontrance qui luy fust faite: la relegation de ces Amans en l'isle de Cadix, où la mère de Meleagre qui les cherchoit aborde et par fortune rencontre Menarque qui estoit à son fils: leur entreveuë heureuse: leur retour en France: la joye et les ceremonies qu'il y eut pour confirmer et approuver leur mariage».

. Diverses variantes (corrections, récritures, quelques additions).

1606 Ars. $8^{\circ}$ BL 20185

Les Amours Diverses divisees en sept Histoires par le Sieur de Nerveze, Secretaire de la Chambre du Roy. Reveuës et corrigees.

Paris, Toussaincts du Bray, 1606 in 12, 400f. + Table et Privilège

- Même privilège, avec même date que pour l'édition de 1605 , mais avec un additif:

«Le dict du Brueil a consenti et consent que Toussaincts du Bray aussi Marchand libraire à Paris jouysse dudict Privilège ainsi qu'il a esté accordé entr'eux ès Estudes des Notaires soubssignez le 28 mai 1605»

. L'ensemble commence par la préface et le sonnet de l'édition de 1605 mais précédé d'une «Epître à Monseigneur le duc de Sully», non foliotée.

- A noter que le nom de Nervèze est suivi d'un nouveau titre; «secrétaire de la chambre du Roi» qui lui reste dans les éditions suivantes. 
- Reprise de l'édition de 1605, avec même foliotation

Sixiesme Histoire: Argument f. 317 et vo. Texte f. 318 (sic 381) - 357 v.

Les retouches et additions au texte de 1602 sont les mêmes qu'en 1605 mis à part trois corrections de détail qui s'y ajoutent et seront maintenues; f. 322: les «discours» de Méléagre ne sont plus «les vrais idoles de la modestie» (1602 f. 14, 1605 f. 322, mais les «vrais patrons»; f. 337 l'adverbe est désormais supprimé dans la formule: «vos desirs osterent infidelement leur masque» $1602 \mathrm{f} .58 \mathrm{v}^{\circ}$ ); 1605 f. 337); f $315 \mathrm{v}^{\circ}$ Méléagre ne dit plus: «nos pueriles ans» mais «nos premiers ans» (1602 f. $101 \mathrm{v}^{\circ} ; 1605$ f. $\left.351 \mathrm{v}^{\circ}\right)$.

$1608 \mathrm{BnF} 8^{\circ} \mathrm{y}^{2} 52756$

Les Amours diverses... Même titre que 1606

Thibaud Ancelin, Lyon, 1608. In 12, $393 \mathrm{f}$.

- Même Privilège du 11 mars 1605 mais avec un autre additif qu'en 1606. Du Brueil en accorde la jouissance à Thibaud Ancelin par un acte daté du 27 juin 1608.

. Pas d'épître à Sully, Préface et sonnet comme en 1606 (avec variantes: Enfans de mes malheurs(...) / Ou mon esprit souloit recueillir ses soupirs...)

. Sixiesme Histoire: Le texte est celui de 1606, sauf pour deux détails où l'on revient à 1602 .

- L'ensemble est suivi de deux «Discours» qui seront publiés à part en 1609: «de la délivrance d'un jeune gentilhomme françois. Autre Discours sur la mort d'une jeune Damoiselle executée dans la ville de Padoue».

1615 Ars. $8^{\circ} \mathrm{NF} 4797$

Les Amours diverses...

Lyon, Barthelemy Ancelin, 1615, in 12, $393 \mathrm{f}$.

. Privilège, daté du 19 mai 1608, à Thibaud Ancelin et «défenses à tous Libraires et Imprimeurs de ce Royaume les imprimer ou faire imprimer vendre ny debiter pendant le temps de six ans entiers et accomplis fors à Anthoine du Brueil...» . Préface et sonnet comme en 1605, mais avec variantes pour le sonnet.

Histoire Sixiesme: Argument f. $311 \mathrm{v}^{\circ}$ Texte f. 312-352.

- Le texte suit celui de 1608, y compris dans les minimes différences par rapport à 1606. Une seule retouche qui n'apparaît pas ailleurs: «l'expérience compatissoit» avec sa jeunesse (1602 f. $73 \mathrm{v}^{\circ}$ et 1605 f. 342) devient «l'experience comparoissoit» (f. 337).

1611 Ars $8^{\circ}$ BL 20186

\section{Les Amours diverses - Dix Histoires}

3 volumes

Les Amours diverses divisees en dix Histoires. Par le Sieur de Nerveze, Secretaire de la chambre du Roy. Reveues et corrigees.

Paris, Toussaincts du Bray, 1611, 3 vol. in 12, 317 f., 286 f., $343 \mathrm{f}$.

. Le Privilège est celui du 11 mars 1605 à Anthoine du Brueil, avec l'additif du 28 mai 1605 le cédant à Toussaincts du Bray (voir édition 1606).

. En tête de l'ensemble, épître non foliotée «A Monseigneur le duc de Sully», comme dans l'édition de 1606 (avec variantes dans l'éloge), préface et sonnet de l'Autheur à son livre

. t. II (avec une épître non foliotée à Monseigneur le Marquis de Rosny).

. Histoire Sixiesme: Argument f. 98 et vºxte, f. 99-153. 
Nombreuses corrections et récritures par rapport au texte de 1606, et deux brèves nouvelles additions.

1617 Sainte Geneviève $8^{\circ}$ Y3894-Inv. 7144 Rés., t. I seulement, Paris, Rolet Boutonné, 1617, Amours diverses...

.t. I in 12 Histoire Sixiesme p. 884-1077. Pas d'epître ni de sonnet mais préface paginée - Argument p. 884. Titre Les Amours de Florigene p. 886. Semble suivre sans corrections la version de 1611.

1621 Poitiers DM 138 - 3 vol. in 12.

Sainte Geneviève (t. II seulement) Y30 895 Inv. 7145 Rés.

Amours diverses...

Rouen, Claude Le Villain, Jacques Aubert et Jean Berthelin .

.t. II au nom de Jean Berthelin, dédicace à M le Marquis de Rosny, dans l'exemplaire de Sainte-Geneviève; dans l'exemplaire de Poitiers, noté I à la reliure, épître à Sully.

Reprise de l'édition de 1611 